\title{
Placing Thermal Ablation for Benign Thyroid Nodules into Context
}

\author{
Roberto Negro ${ }^{a}$ Pierpaolo Trimboli ${ }^{b, c}$

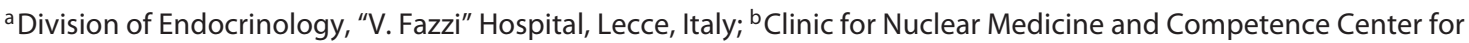 \\ Thyroid Diseases, Imaging Institute of Southern Switzerland, Ente Ospedaliero Cantonale, Bellinzona, Switzerland; \\ 'Biomedical Sciences, Università della Svizzera Italiana (USI), Lugano, Switzerland
}

In the present issue of European Thyroid Journal, Papini et al. [1] published guidelines for the use of thermal ablation (TA) in benign thyroid nodules, sponsored by the European Thyroid Association (ETA). The guidelines highlight the state-of-the-art in TA, clearly define the standard technical procedures, and the indications for which such techniques should be routinely used.

$\mathrm{TA}$, foremost represented by laser and radiofrequency ablation, are minimally invasive techniques predominantly used to reduce the volume of large nodules. Currently there is much more limited experience with microwave and high-focused ultrasound techniques. Well established data from the literature demonstrate that a significant shrinkage can be obtained in the vast majority of treated nodules, accompanied with relief or resolution of cosmetic concerns and compressive symptoms and a consequent improvement of health-related quality of life $[2,3]$. TA, preceded by percutaneous ethanol injection, may be also used to treat large relapsing thyroid cysts and autonomously functioning nodules, alone or in combination with radioiodine [4-6]. The present guidelines are of particular significance, as they offer evidence-based recommendations for clinicians who are already confident with TA, for those who are considering its use, as well as for those who barely know what TA is. Indeed, the ETA survey published in the same issue, clearly shows that many European endocrinologists are not at all familiar with minimally invasive interventional treatments. This surprising result concerns not only "new" techniques, such as laser and radiofrequency ablation, but also better established procedures, such as percutaneous ethanol injection for thyroid cysts [7]. This survey confirms the need for the European guidance that sheds a light on a promising field that is in continuous development. What is more, these are the first guidelines endorsed by an endocrine society that is devoted to the thyroid field: the only other guidelines currently available being from the Korean Society of Radiology [8]. As stated in the present guidelines, TA is primarily suited to management of symptomatic nodules, where the usual standard care is surgery. Indeed, compared to surgery, TA is cheaper, has minor side effects, which are usually transient, is an outpatient procedure, is less time-consuming, and displays a lower rate of hypothyroidism [9]. Symptomatic thyroid nodules also have a significant resource impact in terms of the number of affected patients, and as a consequence, in terms of the number of surgical interventions. TA is thus highly relevant, given that it might potentially avoid about one quarter of thyroid surgical procedures currently performed for large benign nodules $[10,11]$.

\footnotetext{
Roberto Negro 
Grounded on the available best evidence, the guidelines still leave opened some questions frequently asked by patients who are candidates for TA: how much will the nodule shrink? Will the result be durable? Will I need further treatment? Might I undergo surgery sooner or later?

Although, initially targeted to patients who refused surgery or who were at elevated surgical risk, a growing amount of evidence over the last 10 years encouraged Italian and Korean groups to suggest TA as a first-line treatment $[8,12]$. Indeed, TA can induce a mean 12-month volume reduction ratio (VRR) of $50-90 \%$, and this result is known to be sustained for up to 5 years [13]. This remarkable result can be obtained in the majority of patients, but an important point is that the extent of nodule reduction may vary widely and cannot be precisely foreseen. After a single-session TA treatment, approximately one third of laser-treated nodules and about $20 \%$ of the radiofrequency treated nodules display a 12-month VRR $<50 \%$, which is defined as a "technical failure" $[13,14]$. This is of importance as the 12-month VRR is related to the risk and rate of regrowth: the lower the 12-month VRR, the higher the risk of recurrence and shorter the time-to-regrowth [15]. TA is an operator-dependent procedure, and the operator's experience which cannot be standardized is of pivotal importance. What can be standardized, as outlined by the present guidelines, are the operational requirements, i.e., patient eligibility and preparation, the procedure and the post-procedural management. Thus, for example, the delivery of an adequate amount of energy, and the procedural correctness, are essential parts of the operator's arsenal that are crucial for the final results. Another point of interest is that thermal treatments have different efficacies in nodules with different phenotypes. While the skill of the operator is variable and the energy that will be delivered cannot be foreseen before the procedure, nodule volume and its texture are elements that should be carefully evaluated. We know for example that it is difficult to ablate very large nodules in only one session, and that, as a consequence, the untreated part tends to regrow over the time [16]. We also know that spongiform nodules with intra-nodular vascularity shrink more that non-spongiform nodules with peripheral vascularity, as in the former the heat spreads better than in the latter [17]. Nevertheless, the prediction of the final result and the convenience of choosing TA instead of surgery, is still totally dependent on the clinical experience of the physician. Clinical features or classifiers enabling the selection of nodules that respond best or inadequately to these treatments are still under investigation. In addition to the traditional ultrasound evaluation, the usage of instruments such as molecular markers involved in cell proliferation, spectral analysis to categorize nodule composition, or artificial intelligence to predict TA efficacy, may in the future enable the physician to accurately define important characteristics of the nodule, other than its volume [18-20]. Indeed, a critical problem that needs further investigations is the recurrence rate. In these patients, there are more uncertainties regarding nodule recurrence than certainties. Available data show a 5 -year cumulative incidence of regrowth of $28 \%$, and a 5 -year cumulative incidence of retreatment of $10 \%$ [13]. On the one hand, we can state that TA is "cost- and riskeffective", and on the other hand, we are still unable to establish the risk of regrowth for an individual patient, or whether the nodule will need a second thermal treatment or definitive surgery in the future. These critical and unresolved issues need to be considered in a frank discussion with each patient in order to take a shared decision that honestly weighs up the pros and cons of any procedure. Studies are still needed to improve the nodule selection and optimize the standard technique, in order to minimize clinical failures. Finally, and most importantly, when TA is evaluated in respect to surgery, long-term RCTs evaluating success rate, costs, HRQoL and drawbacks, are still needed. This is a decisive point that will help to understand the ultimate role of TA, as to whether it should be considered as a background option or the mainstay of therapy. As we are living in the era of precision medicine, when everyone calls for a tailored treatment, there is no doubt that TA, which is already an extraordinary weapon in the arsenal of thyroid nodule management, needs the solid foundation displayed by the present guidelines. On such foundations we can go further.

\section{Disclosure Statement}

R.N. and P.T. have no conflicts of interest to declare.

\section{Funding Sources}

No funding was received.

\section{Author Contributions}

R.N. and P.T. were equally responsible for drafting the manuscript. 


\section{References}

1 Papini E, Monpeyssen H, Frasoldati A, Hegedüs L. European Thyroid Association Clinical Practice Guideline for the Use of ImageGuided Ablation in Benign Thyroid Nodules. Eur Thyroid J. 2020;2020: https://doi. org/10.1159/000508484.

2 Trimboli P, Castellana M, Sconfienza LM, Virili C, Pescatori LC, Cesareo R, et al. Efficacy of thermal ablation in benign non-functioning solid thyroid nodule: A systematic review and meta-analysis. Endocrine. 2020 Jan; 67(1):35-43.

3 Valcavi R, Tsamatropoulos P. HEALTH-RELATED QUALITY OF LIFE AFTER PERCUTANEOUS RADIOFREQUENCY ABLATION OF COLD, SOLID, BENIGN THYROID NODULES: A 2-YEAR FOLLOW-UP STUDY IN 40 PATIENTS. Endocr Pract. 2015 Aug;21(8):887-96.

4 Døssing H, Bennedbæk FN, Hegedüs L. Interstitial laser photocoagulation (ILP) of benign cystic thyroid nodules-a prospective randomized trial. J Clin Endocrinol Metab. 2013 Jul;98(7):E1213-7.

5 Negro R, Greco G. Quality of life and outcomes in patients with a large toxic adenoma undergoing laser ablation plus radioiodine vs lobectomy. Int J Hyperthermia. 2019 Dec; 36(1):191-5.

6 Cesareo R, Palermo A, Benvenuto D, Cella E, Pasqualini V, Bernardi S, et al. Efficacy of radiofrequency ablation in autonomous functioning thyroid nodules. A systematic review and meta-analysis. Rev Endocr Metab Disord. 2019 Mar;20(1):37-44.

7 Hegedüs L, Frasoldati A, Negro R, Papini E. European Thyroid Association Survey on Use of Minimally Invasive Techniques for Thyroid Nodules. Eur Thyroid J. 2020; https:// doi.org/10.1159/000506513.
8 Kim JH, Baek JH, Lim HK, Ahn HS, Baek SM, Choi YJ, et al.; Guideline Committee for the Korean Society of Thyroid Radiology (KSThR) and Korean Society of Radiology. 2017 Thyroid Radiofrequency Ablation Guideline: Korean Society of Thyroid Radiology. Korean J Radiol. 2018 Jul-Aug;19(4): 632-55.

9 Papini E, Pacella CM, Hegedus L. Diagnosis of endocrine disease: thyroid ultrasound (US) and US-assisted procedures: from the shadows into an array of applications. Eur J Endocrinol. 2014 Mar;170(4):R133-46.

10 Mathonnet M, Cuerq A, Tresallet C, Thalabard JC, Fery-Lemonnier E, Russ G, et al. What is the care pathway of patients who undergo thyroid surgery in France and its potential pitfalls? A national cohort. BMJ Open. 2017 Apr; 7(4):e013589.

11 Sun GH, DeMonner S, Davis MM. Epidemiological and economic trends in inpatient and outpatient thyroidectomy in the United States, 1996-2006. Thyroid. 2013 Jun;23(6): 727-33.

12 Papini E, Pacella CM, Solbiati LA, Achille G, Barbaro D, Bernardi S, et al. Minimally-invasive treatments for benign thyroid nodules: a Delphi-based consensus statement from the Italian minimally-invasive treatments of the thyroid (MITT) group. Int J Hyperthermia. 2019;36(1):376-82

13 Bernardi S, Giudici F, Cesareo R, Antonelli G, Cavallaro M, Deandrea $M$, et al. Long-term results of radiofrequency and laser ablation on benign thyroid nodules: a multicenter study from the Italian minimally-invasive treatments of the thyroid (MITT) group. Thyroid. 2020. Forthcoming.
14 Cesareo R, Pacella CM, Pasqualini V, Campagna G, Iozzino M, Gallo A, et al. Laser Ablation Versus Radiofrequency Ablation for Benign Non-Functioning Thyroid Nodules: SixMonth Results of a Randomized, Parallel, Open-Label, Trial (LARA Trial). Thyroid. 2020 Jun;30(6):847-56.

15 Negro R, Greco G, Deandrea M, Rucco M, Trimboli P. Twelve-month volume reduction ratio predicts regrowth and tome to regrowth in thyroid nodules submitted to laser ablation: a 5-year follow-up retrospective study. Korean J Radiol. 2020 Jun;21(6):764-72.

16 Sim JS, Baek JH, Lee J, Cho W, Jung SI. Radiofrequency ablation of benign thyroid nodules: depicting early sign of regrowth by calculating vital volume. Int J Hyperthermia. 2017 Dec; 33(8):905-10.

17 Deandrea M, Garino F, Alberto M, Garberoglio R, Rossetto R, Bonelli N, et al. Radiofrequency ablation for benign thyroid nodules according to different ultrasound features: an Italian multicentre prospective study. Eur J Endocrinol. 2019 Jan;180(1):7987.

18 Liu H, Lin F. Application of immunohistochemistry in thyroid pathology. Arch Pathol Lab Med. 2015 Jan;139(1):67-82.

19 Granchi S, Vannacci E, Biagi E. Characterization of benign thyroid nodules with HyperSPACE (Hyper Spectral Analysis for Characterization in Echography) before and after percutaneous laser ablation: a pilot study. Med Ultrason. 2017 Apr;19(2):172-8.

20 Negro R, Rucco M, Creanza A, Mormile A, Limone PP, Garberoglio R, et al. Machine learning prediction of radiofrequency thermal ablation efficacy: a new option to optimize thyroid nodule selection. Eur Thyroid J. 2020; https://doi.org/10.1159/000504882. 